\title{
"Soy una persona que no existe": derechos y ciudadanía en las trayectorias de brasileños y brasileñas sin documentos
}

\author{
Fernanda da Escóssia \\ IBMEC Rio \\ fernanda.daescossia@uol.com.br
}

Recibido: 16/7/2018 / Aceptado: 1/10/2018

doi: 10.26439/contratexto2018.n030.3152

\begin{abstract}
Resumen. El artículo examina de qué forma los brasileños adultos indocumentados - hombres y mujeres - buscan el primer documento de su vida, el acta de nacimiento, en un servicio gratuito instalado por el Tribunal de Justicia del Estado de Río de Janeiro dentro de un autobús en el centro de la ciudad. El texto hace un recorrido histórico del sistema del Registro Civil en Brasil y presenta el tema del subregistro del nacimiento enfocado en los adultos. Describe el campo y la metodología utilizada, y recurre a la observación participante a partir de las historias de vida de dos mujeres. El texto discute conceptos como la identidad y los derechos, expresados por los brasileños sin documentación, y reflexiona sobre el papel del documento como clave para el acceso a la ciudadanía. A partir de la obra de Bourdieu, el trabajo analiza el certificado de nacimiento como resultado de un rito de institución y problematiza el significado atribuido al documento por los usuarios. El artículo es el resultado de dos años de trabajo etnográfico de la autora e incluye parte de la investigación realizada para su tesis de doctorado. Fue presentado en el marco de la 7. Reunión Mundial de Cátedras Unesco, en el Congreso Internacional "Comunicación, ciudad y espacio público", celebrado en Lima en mayo del 2018.
\end{abstract}

Palabras clave: registro de nacimiento / documentación / derechos / ciudadanía 


\title{
"I am a person who does not exist": rights and citizenship in the trajectories of undocumented Brazilians
}

\begin{abstract}
Авsтract. This article examines how male and female undocumented Brazilian adults seek the first document of their lives, the birth certificate, in a free service set up by the Court of Justice of the state of Rio de Janeiro inside a bus in the center of the city. This paper presents the history of the Brazilian civil registry system and the birth under-registration focused on adults. It describes the field and methodology used, and examines the participants based on life stories of two women. It discusses concepts such as identity and rights expressed by undocumented Brazilians and reflects on the role of the document as a key to access to citizenship. Based on Bourdieu's work, the article analyzes the birth certificate as a result of an institutional rite and problematizes the meaning attributed to the document by users. The research is the result of two years of the author's ethnographic work, which includes part of the research she carried out for her PhD thesis. It was presented at the 7th World Meeting of UNESCO Chairs in Communication, at the International Symposium "Communication, City and Public Space", held in Lima in May 2018.
\end{abstract}

Keywords: birth certificate / documents / rights / citizenship 


\section{Introducción}

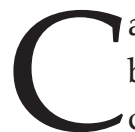

ada viernes, el autobús azul y blanco estacionado en el patio de la Vara de la Infancia y de la Juventud, en la Plaza Once, en el Centro de Río de Janeiro, se agita con el entra y sale de gente a partir de las 9 de la mañana. En el exterior, nunca menos de cincuenta personas, todas pobres o muy pobres, casi todas negras, rodean el vehículo, preguntan, se sientan y se levantan, preguntan de nuevo y esperan sin reclamar el tiempo que sea necesario. Adultos, ancianos y niños están allí para conseguir el primer documento oficial del ciudadano brasileño: el certificado de nacimiento.

El autobús de la Plaza Once es resultado de una asociación entre dos proyectos del Tribunal de Justicia del Estado de Río de Janeiro (en adelante, TJRJ), la Justicia Itinerante y el Servicio de Promoción de la Erradicación del Subregistro de Nacimiento y la Búsqueda de Certificados (en adelante, Sepec); es el corazón de un proyecto mantenido por el TJRJ con el fin de reducir el llamado subregistro, es decir, la proporción de personas sin certificado de nacimiento. Es también el objeto de la investigación de doctorado que da origen a este texto. La pesquisa parte del trabajo de Peirano (2006), “¿De qué sirve un documento?”, y dialoga con la línea de pensamiento que reflexiona acerca del documento como instancia de control (Foucault, 2015; Carrara, 1984) y, al mismo tiempo, de aceso a la ciudadanía (DaMatta, 2002; Peirano, 2006; Vianna, 2013; De Mesquita
Ferreira, 2015). Estudios más recientes ayudan, por fin, a pensar el documento como artefacto central de las burocracias modernas (De Mesquita Ferreira, 2013; Schritzmeyer, 2015; Pinto, 2014, 2016).

En este artículo se discute cómo la búsqueda del certificado de nacimiento saca a la luz concepciones de identidad, derechos y ciudadanía expresados por los brasileños adultos que llegan al autobús de la Plaza Once. Se analiza también la manera en que ellos se presentan como sujetos de derechos y de qué modo sus nociones de identidad, derechos y ciudadanía se modifican; es decir, de qué manera se transforma quien busca este documento: el acto de recibir el registro de nacimiento después de años sin identificación legal es valorado por los usuarios como la puerta hacia el reconocimiento de sí mismos en tanto titulares de derechos hasta entonces negados, y como medio para la reconstitución de trayectorias familiares.

El artículo justifica la opción metodológica, la etnografía, pues permite valorizar la experiencia y las vivencias de los usuarios en diálogo con estudios de antropología del Estado y antropología del derecho. Este enfoque ayuda a comprender el Estado y sus márgenes (Sharma y Gupta, 2006; Vanegas, 2016) en dos dimensiones: las prácticas cotidianas (las rutinas, los procedimientos burocráticos que a menudo parecen banales) y las representaciones de este, o sea, las formas que él asume y a través de las cuales se presenta. 
Etnografiar a una persona adulta sin documento es sumergirse en lo que Das y Poole categorizan como márgenes del Estado (2004), "los lugares a partir de los cuales intentamos entender lo que cuenta en el estudio del Estado en la antropología" (p. 3); es decir, las prácticas, los lugares y los lenguajes existentes en espacios que parecen estar en los límites del funcionamiento regulado del Estadonación, entendido como la presencia que da la estructura al sentido y las formas del poder en cualquier sociedad. Das y Poole (2004), Telles (2010) y Brulon y Peci (2013) llevan a reflexionar sobre las relaciones que se desarrollan en esos márgenes, tradicionalmente percibidos como áreas en las que el Estado no está presente, aunque sí lo esté, solo que de modo no regular ni regulado. Los márgenes en mención constituyen parte del Estado, pues este también se hace en los márgenes (Das y Poole, 2004). Otra reflexión necesaria es sobre cómo tales márgenes -muchas veces entendidos como áreas en las cuales el Estado fue incapaz para imponer orden, lugares donde solo habría exclusión y desordenreorganizan sus prácticas y experiencias en una construcción que no es monolítica, sino procesal y dinámica. En los márgenes del Estado, la observación etnográfica de esas prácticas y vivencias muestra que la exclusión y el desorden conviven con resistencia y pluralidad.

¿Cómo vive un adultosin documentos en una sociedad documentada? ¿Cómo se ve sin documentos? ¿Qué papel atribuye al registro de nacimiento? "Quiero el registro de nacimiento para inscribirme en el programa Bolsa Familia" ${ }^{1 "}$, decía uno. "Quiero el registro de nacimiento para sacar el carnet de trabajo", afirmaba otro. "Quiero el registro de nacimiento para poder entrar en la jubilación", aclaraba el tercero. "Quiero el registro de nacimiento para colocar a mi hijo en la escuela", resaltaba en la habitación. Las respuestas de los usuarios del autobús de la Plaza Once indicaban inicialmente que el registro de nacimiento tendría finalidad inmediata, pues ellos decían querer el documento para conseguir otra cosa, muchas veces otro documento, acceso a políticas públicas, beneficios o servicios.

Sin embargo, a lo largo de dos años de investigación de campo en el autobús de la Plaza Once, se pudo observar que el registro de nacimiento no tiene solo una finalidad inmediata. Los relatos obtenidos durante el trabajo de campo indican que, en el proceso de obtención del documento, muchos usuarios -hombres y mujeres- buscan reconstruir su propia historia y recuperar lazos familiares en un proceso que Schritzmeyer (2015) llama recuperación de los "hilos de sus vidas". En la búsqueda de documentación, la dimensión inmediata e innegable de "para qué sirve el registro de nacimiento" se une a otra, que remite a la otra búsqueda, la de los derechos, el acceso a la ciudadanía y la recuperación

1 Programa de transferencia de renta del gobierno brasileño. 
de la propia historia familiar. El presente artículo trae parte de las reflexiones que surgieron durante la construcción de la tesis de doctorado en el Centro de Pesquisa y Documentación de Historia Contemporánea de Brasil (CPDOC), unidad de la Fundación Getulio Vargas (FGV). De julio a diciembre del 2017, la investigación tuvo el inestimable apoyo financiero de la Escuela de Derecho de la FGV, institución a la que la autora del presente artículo quedó afiliada como investigadora fellow.

No hay tiempo ni espacio para ver aquí la revisión de la literatura utilizada en la tesis acerca de la historia del registro de personas y su asociación con el tema de los derechos y la ciudadanía (DaMatta, 2002; Caplan y Torpey, 2001; Bourdieu, 1996b; Carrara, 1984; Brasileiro, 2008; Hunter y Sugiyama, 2017). El registro de personas se remonta a la antigüedad, aunque inicialmente la finalidad fue militar y tributaria, indica DaMatta (2002). Brasileiro (2008) recuerda que, en la Grecia antigua, los padres pedían la inscripción de sus hijos e hijas en el libro de los ciudadanos libres. Dos siglos antes de Cristo, había un registro civil en China. Los incas tenían un sistema de anotaciones de nacimientos y muertes. En el siglo XvI, el Concilio de Trento hizo obligatorios los registros parroquiales con datos de nacimientos, bodas y muertes. DaMatta (2002) sitúa en el siglo xviI el punto probable de ese monitoreo continuo de la población, y lo relaciona a la ola de modernización de la que la Revolución francesa es el gran hito.
Makrakis (2000) recuerda que, históricamente, en el Brasil colonial, debido a la fuerte conexión entre la Iglesia y el Estado, los registros de las parroquias tenían valor de prueba $\mathrm{y}$ perduraron como forma de identificación. El registro civil de nacimiento solo se convirtió en acto del Estado en la República, con la separación de Iglesia y Estado. Paralelamente a la institución del registro civil laico, el Imperio de Brasil concedió a las instituciones carcelarias privadas la función de efectuar los registros. Desde el imperio, las oficinas funcionaban bajo el régimen de concesión gubernamental, los cargos de sus dirigentes (los notarios) eran vitalicios y podían hacer un traspaso hereditario de sus funciones a sus sucesores.

El régimen de concesión gubernamental para explotación de la actividad carcelaria, practicado desde la época imperial, creó puestos vitalicios para sus dirigentes y estableció una especie de derecho de la herencia para las vacantes de sus sucesores, además de haber propiciado verdaderos monopolios de regiones y ciudades con solo una oficina de registro civil, teniendo en cuenta que las concesiones eran esporádicas y segmentadas. Se creó así, en Brasil, un sistema atípico y peculiar que perdura por más de un siglo. (Makrakis, 2000, pp. 32-33)

La Constitución brasileña de 1988 prohibió el nombramiento de los dirigentes de los notarios por libre elección del gobernante y previó la realización de concursos públicos para llenar las vacantes. El sistema de registro civil está regulado en Brasil por la Ley 6.015, 
del 31 de diciembre de 1973. De acuerdo con esta ley, el registro de nacimiento se debe realizar en el Registro Civil de Personas Naturales (RCPN), entidad privada que ejerce la función por delegación del poder público. El registro debe ser hecho por los padres hasta quince días después del nacimiento del niño, mediante la presentación de documentos y de la Declaración de Nacido Vivo (DN) emitida por el hospital; no obstante, el plazo de registro se podrá prorrogar hasta tres meses. La oficina expedita el certificado de nacimiento; por la ley 9.534, de diciembre de 1997, la primera vía del documento es gratuita para el ciudadano. Los papeles reciben el pago de las Corregidoras de Justicia, vinculadas a los Tribunales de Justicia de los Estados (Instituto Brasileiro de Geografia e Estatística, 2015).

Por las leyes del Estado brasileño, el registro civil es el acto legal que certifica la existencia de alguien y genera el primer documento oficial, el certificado de nacimiento. DaMatta (2002) analiza el sistema brasileño como encadenado, pues para obtener un documento es siempre exigido uno anterior; también menciona documentos centrales y periféricos, y cita como documento fundador el certificado de nacimiento. Después de este, vienen otros documentos, como el número de identificación ante la Fiscalía $(\mathrm{CPF})$, el carnet de conducir, el certificado de reservista (que los hombres reciben al prestar servicio militar), el título de elector, el carnet de trabajo, el pasaporte y el certificado de defunción. Quien no tiene documentación es enterrado como indigente, en sepultura sin identificación.

A inicio de la década del 2000, quien suscribe este artículo, todavía como periodista, empezó a escribir acerca del tema del registro de nacimiento. A pesar de la ley de la gratuitidad de este acto, en el 2002, datos oficiales del Instituto Brasileño de Geografía y Estadística (en adelante, IBGE) monstraban el porcentaje de subregistro en 20,3\% (nombre técnico para el fenómeno de niños sin registro de nacimiento). El subregistro es definido por el IBGE como el conjunto de nacimientos no registrados en el año de ocurrencia o hasta el final del primer trimestre del año siguiente. La estimación se calcula por la diferencia entre los nacimientos estimados y los informados por los registros (IBGE, 2015).

Ante esta realidad, a partir del 2003 se inició, con la coordinación de la Secretaría de Derechos Humanos de la Presidencia de la República, durante el gobierno del presidente Luiz Inácio Lula da Silva (Partido de los Trabajadores), un movimiento de reducción del subregistro, con la creación de comités en el ámbito federal, de los estados y de los municipios para implementar acciones contra el problema. En el 2007, el Gobierno brasileño lanzó el Compromiso Nacional por la Erradicación del Subregistro Civil de Nacimiento y la Ampliación del Acceso a la Documentación Básica, un programa nacional con la participación de los estados y de los municipios, además de órganos del gobierno y de entidades de justicia. Este plan 
estableció como documentación básica el Catastro de Personas Físicas (CPF), el Carnet de Identidad o Registro General (RG) y el Carnet de Trabajo y Previsión Social (CTPS). En el 2014 se instituyó el SIRC (Sistema Nacional de Información de Registro Civil) con el objetivo de captar informaciones de registros de nacimiento, matrimonio y muertes generadas por los registros y almacenarlos en una base centralizada que permita formular políticas públicas (Garrido y Leonardos, 2017).

El censo (recenseamento) del 2010, el último realizado en Brasil, contabilizó 599000 niños menores de diez años sin registro de nacimiento, el $2 \%$ del total de este grupo de edad (IBGE, 2015). En el 2015, según las estadísticas de Registro Civil producidas anualmente por el IBGE, el porcentaje de subregistro de niños había caído al 3,2 \% (IBGE, 2015), y la experiencia brasileña pasó a constar, con referencias elogiosas, en los informes de las Naciones Unidas. En un análisis del resultado del programa brasileño de combate contra el subregistro, Hunter y Sugiyama (2017) apuntan a la implementación de políticas de transferencia de renta - entre ellas, los programas Bolsa Familia y Beneficio de Prestación Continuada (BPC) - como decisiva para la reducción del problema, pues, para ser atendidas por las políticas sociales, las familias necesitaban tener a todos los integrantes documentados. Durante la investigación de campo, el Bolsa Familia, programa de transferencia de ingresos implementado en el 2003, aparece en las entrevistas como uno de los motivos más citados por los usuarios para obtener el registro de nacimiento. Más difícil, casi imposible, ha sido obtener estadística sobre los adultos sin documentación, ya que no figuran en ninguna base de datos específica. Como no existen oficialmente, no pueden ser contados. El IBGE no tiene una estimación del total de hombres y mujeres sin documentación en Brasil.

Mucho se habla de problemas de documentación para imigrantes. Dentro de esos estudios, es ventajosa la línea de investigaciones acerca de la representación discursiva de imigrantes en los medios (Hernández, 2010; Van Dijk, 2006; Matínez Lirola, 2008, 2014, 2017). Destaco que la presente investigación, entretanto, tiene como foco a brasileñas y brasileños sin documentos dentro de su propio país, en una condición que se asocia a la pobreza y a las dificultades con las instancias estatatales. No son imigrantes, son brasileñas y brasileños, y esta es una de las contribuciones que desde este texto se desea ofrecer a la formulación de políticas públicas acerca del tema.

\section{Metodología}

Esta sección presenta la etnografía como metodología de trabajo y describe el campo de estudio. Se optó por ella, con observación participante, porque permite reconstituir las experiencias de las personas en su vida indocumentada, así como comprender las dinámicas de atención del autobús. Becker señala que la observación participante tiene el mérito de abordar a las personas 
"enredadas en relaciones sociales que son importantes para ellas" (1997, p. 75), y son justamente esas relaciones las que le interesa conocer al científico social.

Para esta investigación, se acompañó el trabajo de la Justicia Itinerante en el autobús durante cuarenta viernes, desde septiembre del 2016 hasta diciembre del 2017. En este periodo, se entrevistó a beneficiarios, jueces, fiscales, defensores, funcionarios. Se anotó y preguntó, pero también se colaboró entregando documentos, apoyando procesos, haciendo copias, dando información, llenando formularios, ofreciendo galletas y agua a los niños, dando recados, y otros. Incluso, un día se ayudó a una mujer semianalfabeta, madre de cuatro hijos, a firmar cuatro veces su propio nombre, copiando cada letra, para solicitar el registro de nacimiento de ella y de los niños.

Toda la investigación fue metodológicamente construida a partir de la observación participante. Se siguió todo el proceso de servicio en el autobús, incluyendo la selección, las entrevistas de los usuarios llevadas a cabo por funcionarios de autobuses, las audiencias con los jueces y el momento en que las personas recibían el certificado de nacimiento en el registro del Juzgado de Infancia y Juventud. Se tuvo muchas conversaciones informales y se pudo observar bastante: se les pedía a las personas que contaran lo que buscaban, cómo habían llegado hasta allí y por qué buscaban el documento, además de otra información, como la edad, renta, escolaridad y ausencia de documentación en la familia. Unas hablaban más, otras menos, pero nadie se negó a responder. Al inicio, se grabaron algunas entrevistas, pero al notar que la gente se sentía un poco intimidada, se optó por anotar todo en cuadernos. Otro elemento que dificultaba las entrevistas grabadas era el alto ruido ambiental, que perjudicaba mucho la calidad del audio.

De septiembre del 2016 a agosto del 2017, se entrevistó a cerca de ochenta personas, entre usuarios del autobús, funcionarios de Justicia Itinerante, jueces, defensores y fiscales. Se grabaron alrededor de 12 entrevistas, se tomaron más de 250 fotos y cerca de 30 videos durante la investigación etnográfica.

\section{En un autobús, un pedazo de un país}

El autobús azul y blanco estaciona en el patio del Juzgado de la Infancia y de la Juventud, en el Centro de Río de Janeiro, alrededor de las 8 de la mañana del viernes. El servicio, iniciado en el 2014, resulta de la asociación de dos proyectos del Tribunal de Justicia de Río de Janeiro: la Justicia Itinerante, que busca facilitar el acceso a la prestación de servicios judiciales, y el Sepec, creado en el marco de la política nacional de reducción del subregistro. A diferencia de otras unidades de la Justicia Itinerante, el autobús de la Plaza Once solo atiende a personas que buscan el certificado de nacimiento.

Lúcia, comisaria de justicia, anota por orden de llegada los nombres de las personas que quieren atención. Como el autobús de la Plaza Once solo emite registro de nacimiento, quien busca otro 
servicio es dirigido a otra dirección. Con esta primera selección comienza otro viernes de los cuarenta en que se acompañó el trabajo de campo, y durante los cuales se tuvo acceso irrestricto al funcionamiento del autobús.

Cada viernes el autobús realiza por lo menos cincuenta llamadas. Los datos obtenidos en el Tribunal de Justicia del Estado de Río de Janeiro muestran que, del 2015 al 2017, el notario de la Justicia Itinerante del Subregistro inició 893 nuevas acciones de personas solicitando el registro tardío. Se emitieron 795 certificados de nacimiento. El número no incluye personas que solicitaron cambio de nombre, segunda vía de la documentación o reconocimiento de paternidad. Se refiere únicamente a personas jamás registradas que obtuvieron el primer documento oficial de sus vidas.

Decidí seleccionar historias de vida que me servirían como hilos conductores en la investigación. A pesar de no ser método exclusivo de la antropología, la historia de vida ofrece, entre sus ventajas, la posibilidad de comprender, en datos biográficos de un individuo, las relaciones entre los miembros de un grupo social, y su fuerza como método crece a partir de los años veinte del siglo pasado (Langness, 1973, p. 27). Para el autor, la expresión historia de vida se utiliza

para expresar los datos a lo largo de la vida de una persona, tanto los relatados por la propia persona como los reportados por otras, o por ambas, e incluso si tales datos fueron escritos o fueron recolectados en entrevistas o en ambos casos. (Langness, 1973, p. 17)

Boas (1943) cuestionó el valor de la historia de vida como herramienta metodológica debido a que las descripciones de algunos informantes solamente representaban parcialmente a un grupo; para él, la historia de vida (aunque útil al permitir analizar cómo comportamientos individuales se relacionan con cambios políticos, religiosos o económicos) puede llevar al error de hacer generalizaciones basadas en declaraciones de un interlocutor. Boas, como Bourdieu haría más tarde, llama la atención sobre piezas que la memoria pliega y apunta algunos de los riesgos de aceptar autobiografías como datos confiables. Para Bourdieu (1996b), la ilusión biográfica nos hace creer que la vida narrada por quien la vive es un conjunto coherente y orientado; esto lo lleva a desconfiar del modelo de la presentación oficial que cada uno hace de sí mismo. El autor atenta contra la necesidad de entender la trayectoria de una persona no como línea única y regular; por el contrario, los acontecimientos biográficos son desplazamientos en el espacio social.

Sin embargo, seguidores fieles de Boas defienden las historias de vida como metodología. Entienden que su valor es justamente mostrar, con trayectorias de personas particulares, cómo determinada cultura actúa en sus vidas. Este es el camino de Mintz (1984), que, en un estudio sobre trabajadores de caña puertorriqueños, combina metodológicamente etnografía e historia de vida, utilizando 
una historia de vida para comprender una situación colectiva. De este autor destaco la reflexión sobre por qué elegir uno $\mathrm{u}$ otro interlocutor. Según él, las razones de la elección son recurrentes entre antropólogos: 1) un informante particularmente competente en describir su cultura; 2) un informante que ya tuvo contactos externos y trabajó con otros antropólogos, y 3) simpatía mutua entre el antropólogo y su informante. Este es el relato de cómo su informante se convirtió en sujeto de la investigación:

La respuesta es más simple de lo que parece: yo no "escogí" a Taso, él me "escogió". Él podría haberme rechazado el primer día en que nos encontramos; pero, en cambio, colaboró conmigo. [...] Él me escogió. Trabajando con él, descubrí más sobre mí mismo. (Mintz, 1984, p. 57)

En esta investigación se ha mezclado la observación participante con historias de vida, en un intento por comprender historias colectivas a partir de vivencias individuales. Los criterios para seleccionar las historias fueron la disponibilidad del interlocutor, la claridad de las informaciones y el diálogo con las categorías trabajadas. Al mismo tiempo, el texto de Mintz recuerda que tal vez no se haya escogido a esas personas: sentimos que ellas nos escogieron, y agradecemos que hayan hablado con nosotros. Por decisión metodológica y protección, se han cambiado los nombres de todas las personas que trabajan en el autobús de la Plaza Once. En cuanto a los usuarios, se les identifica solo por el nombre, pues encontramos injusto llamar de forma diferente a quien tardó tanto para obtener un documento con su propio nombre.

\section{Resultados}

En esta sección se presentan los resultados de la investigación etnográfica, con énfasis en las representaciones que las personas sin documentos hacen de sí mismas como sujetos de derechos, y se analiza cómo se interpretan. El recurso de dos historias de vida permite adentrarse en las trayectorias de dos mujeres, Cristiane y Rita.

\section{¿Quién soy cuando no tengo documentos?}

El primer caso del viernes 21 de octubre del 2016 es una audiencia de una familia de mujeres negras en la que tres generaciones no tienen registro de nacimiento: Cristiane, de 36 años; su hija Krícia, de 19 años, y su nieta Mayra, de 2 años, hija de la joven. La familia vive en Belford Roxo, municipio de Baixada Fluminense, y llegó al autobús gracias a la Fundación Municipal de Desarrollo Social de Belford Roxo (Fumbel). Cristiane cuenta que necesita registrarse para registrar a su hija y que luego ella pueda registrar a la nieta. “¿Por qué?", insistimos. "Quiero el Bolsa Familia para mi nieta. Nunca solicité los documentos, pero ahora es urgente", resume Cristiane. Su madre tuvo 17 hijos, Cristiane cuenta en su entrevista que no conoció a su padre y que su madre, que no estaba en condición de criar a sus hijos, los distribuyó 
entre parientes. Cristiane fue "dada" para vivir con una tía. No se registró nunca. Cuando volvió a vivir con su madre, ya en la adolescencia, las dos fueron a la oficina, pero esta no aceptó registrar a la adolescente. Una vez, Cristiane le preguntó a su madre por qué no tenía el acta de nacimiento:

Ella no supo decirme. Me quedé molesta, pero creo que eran muchos hijos... Ella no tuvo responsabilidad. Cuando ella murió, vi que su acta de muerte no tiene nombre. Ella tampoco tenía documento, ¿verdad? Mi padre registró a los hijos mayores, pero yo no.

Cristiane concluyó la enseñanza fundamental y comenzó a trabajar como empleada doméstica para ayudar a su madre, también doméstica. Nunca le faltó trabajo, pero tampoco apareció un patrón que la ayudara a sacar la documentación. Cuando la conocí, Cristiane trabajaba como cuidadora de ancianos y ganaba 1800 reales mensuales, sin contrato firmado. La falta de registro de nacimiento era obstáculo para obtener otros documentos, como el CPF, el título de elector o el carnet de identidad. Nunca había votado. Pagaba 450 reales de alquiler. Tenía empleo y renta fijos, raro entre los usuarios del autobús. Ya estaba separada del padre de su hija.

Krícia, la joven, no trabajaba fuera, sino que se quedaba cuidando a Mayra y la casa. Observo que su brazo derecho está quemado. "Fue su padre" - dice Krícia, apuntando a la niña-, "que me quemó con la plancha para alisar el pelo". Ella conoció al muchacho en un baile, quedó embarazada y tuvo un niño, Enzo, que murió una hora después de su nacimiento. Ese mismo viernes, 21 de octubre, entrevisté a Krícia. Cuando le pregunté la causa de la muerte de su niño, ella contestó lo siguiente:

No sé muy bien, no... El embarazo fue mal porque su padre me golpeaba todos los días. A menudo, no recuerdo cuántas veces. Él me pateaba en la barriga, golpeaba a mi madre también. Él estaba involucrado... Robaba coches, motos, entró en el tráfico. Fue arrestado una vez. Nos separamos, pero volvimos y quedé embarazada de nuevo. Antes de que naciera, él me mandó y volví a casa de mi madre.

\section{$[\ldots]$}

Un día, él me vio en la calle con el pelo ordenado y me dijo que el pelo liso no era para mí. Tomó la tabla y me quemó. Entonces me dije a mí misma que no lo quería más, y se lo dije a él también. Entonces me golpeó de nuevo; lo denuncié a la policía, pero no pasó nada...

Le pregunto sobre la falta de documentos y Krícia responde que lamenta no haber estudiado, pues en la escuela, después de las series iniciales, le exigían documentación:

Yo ya tuve una oportunidad de trabajo y la perdí porque no tenía documentos. Si tuviera documentos, yo querría ser arquitecta, médica o estudiar enfermería... Pero no desistí del todo, todavía tengo mis derechos.

La historia de vida de Cristiane permite reflexionar sobre categorías fundamentales en esta investigación: derechos y ciudadanía. Tema de convenciones mundiales, tratados 
internacionales y compendios, los derechos son una categoría ampliamente tratada en las ciencias sociales y jurídicas, dentro de variados matices teóricos y metodológicos. El diálogo de esta investigación con los derechos, sin embargo, no se dará a partir de la definición legal, jurídica, histórica o filosófica del concepto, sino a partir de otra clave, que los privilegia como vivencias y experiencias, y que evidencia la dimensión simbólica y discursiva de los derechos, en plural. Schritzmeyer (2012), Vianna (2013), Souza Lima (2012) y De Mesquita Ferreira (2013) usaron esta perspectiva en la observación etnográfica de experiencias y vivencias de quienes buscan derechos ante instancias del Estado. No se trata, como alerta Vianna, de menospreciar el marco legal, sino de "tomarlo en diálogo con usos, contradicciones y conflictos motivados en la idea misma de que hay algo que sea 'los derechos'" (2013, p. 16).

\section{Souza Lima destaca:}

Los derechos surgen como categoría asociada a lo que llamaré comunicación entre esferas sociales, esferas que tradicionalmente surgen separadas. Las emociones, los sentimientos y los afectos circulan y se entrelazan en instituciones como la Policía, el Legislativo o instancias del Poder Judicial; en suma, con lo que también los actores sociales concretos llaman Estado, aquí no solo tomado como concepto científico, sino también como categoría de los encuestados hablados y articulados a través de la lucha por los derechos [cursivas de la autora]. (Souza Lima, 2012, p. 12)
El diálogo con la antropología del derecho enfatiza la elección analítica para esta investigación: la comprensión de cómo y por qué la documentación se inserta en el campo de los derechos, investigando el impacto de la ausencia de la documentación sobre el ejercicio de estos mismos. En ese marco, el enfoque propuesto por Vianna (2013) afirma que su objetivo es tratar la dimensión de acción social de los derechos en tanto norma, tradición administrativa o forma de la construcción de sujetos morales y políticos, así como la dimensión procesal y dinámica. También indica que, más que definir el concepto jurídico de los derechos, la propuesta es tomarlos en diálogo con los usos, las contradicciones y los conflictos presentes en el ejercicio cotidiano de estos. La legislación, los aparatos judiciales y burocráticos, y las instituciones son propicios para la realización de estudios etnográficos interesados en abordar disidencias, contradicciones, silencios, estrategias accionadas de modo personal o colectivo frente a la letra de la ley, sea el mostrador de la comisaría o el autobús de la Justicia Itinerante.

Atravesando y transitando entre dominios que pueden ser clasificados por los propios actores como políticos, judiciales, administrativos o de políticas públicas, el lenguaje de los derechos reafirma disensiones morales, ofreciendo espacio para que sean tejidas contranarrativas y para que nuevos sujetos se hagan presentes en escenas públicas. (Vianna, 2013, p. 16)

Schritzmeyer (2012) afirma que es necesario extrañar "el aparente consenso 
que ronda los términos derecho, derechos y justicia" (p. 264). El consenso sobre los derechos no se da previamente, sino que se disputa y se negocia, incluso cuando se habla de un derecho garantizado por la legislación nacional e internacional. Szreter (2007) sostiene que la identificación es un derecho humano, el "derecho de tener una identidad legal y sus relaciones con otras personas públicamente reconocidas, seguramente registradas y accesibles para su uso personal" (p. 67). El Pacto Internacional de Derechos Civiles y Políticos, adoptado por la XXI Sesión de la Asamblea General de las Naciones Unidas el 16 de diciembre de 1966, establece en su artículo 24, párrafo, 2 que todo niño deberá ser registrado inmediatamente después de su nacimiento y deberá recibir un nombre. Solo con el fin de la dictadura militar y tras la promulgación de la Constitución de 1988, Brasil se adhirió al Pacto Internacional.

Sin consensos previos, la reflexión sobre la búsqueda del registro de nacimiento se sitúa en el debate sobre el acceso a los derechos y la justicia en Brasil, problematizando la forma en que las personas sin documentación se insertan en una sociedad documentada, qué idea tienen de sí mismas y de qué modo se presentan como merecedoras del derecho de tener el documento. Tomemos inicialmente la afirmación de Cristiane, presente en varias entrevistas con el mismo tono vago: también quiero mis derechos. Para cobrar derechos -explicitando el entendimiento de que, por tanto, se percibe como sujeto de derechos-, Cristiane, como otros usuarios del autobús, se presenta discursivamente como alguien destituido de derechos.

La historia de Cristiane puede ser entendida como una continua negación de derechos, pues la falta de registro alarga el camino para obtener otros derechos, documentos, escuela, atención médica. Al decir que busca sus derechos, Cristiane relata su experiencia de vida repetidamente carente no solo de derechos, sino del derecho a tenerlos. Sin registro de nacimiento, otros derechos le fueron negados, como el empleo formal y el voto. Cristiane habla de sí como alguien que, por no tener registro de nacimiento, perdió otros derechos. Este relato se repite en numerosos interlocutores, como muestran los extractos de entrevistas a personas atendidas en el autobús:

- "La persona, cuando no se registra, queda como un nadie, la persona no existe." (Carlos, 22 años)

- "Me siento una nada, la gente no existe." (Fátima, 57 años)

- "Me siento un perro. Soy una persona que no existe" (María, 52 años)

- "Soy un cero a la izquierda" (Reginaldo, 63 años)

El discurso de los usuarios - "cero a la izquierda", "perro", "una nada", "persona que no existe"- contiene expresiones que muestran claramente la idea que la persona sin registro de 
nacimiento tiene sobre sí misma: una persona sin valor, cuya existencia nunca fue oficialmente reconocida; una persona, por tanto, que no se constituyó en sujeto de derechos. Peirano (2006) define el documento como algo que identifica al individuo para fines de cesión de derechos y cobranza de deberes, al mismo tiempo que analiza el precio de la ausencia de documentación: la negación de la existencia de aquel individuo como ciudadano.

El documento, así, legaliza y oficializa al ciudadano y lo hace visible, pasible de control y legítimo para el Estado; el documento hace al ciudadano en términos performativos y obligatorios. Esta obligatoriedad legal de poseer documentos naturalmente tiene su lado inverso: el de quitar, despossuir, negar y vaciar el reconocimiento social del individuo que no posee el documento exigido en determinados contextos. (Peirano, 2006, pp. 26-27)

La historia de vida de Cristiane explicita cómo ella, para presentarse como sujeto de derechos ("yo también tengo derechos"), al mismo tiempo se desconstruye al decir que no es nadie, de igual forma como otros usuarios se presentaron como "una nada", un "perro" o "una persona que no existe". Sin documento, el individuo vacía el reconocimiento de sí mismo como merecedor de derechos para luego volver a pleitearlos a partir de la obtención del registro civil. Al verbalizar el hecho de no ser nadie, o no existir -aunque, naturalmente, sea alguien-, la persona sin documentos explicita el modo cómo ve al Estado y cómo se ve a sí misma ante dicho ente, que es una idea transmutada de muchos modos en la definición de Abrams (2006).

Este autor distingue el estado-sistema del estado-idea y afirma que mientras el primero se caracteriza por sus prácticas y su estructura, el segundo -la administración en sí- es reificado y gana entidad abstracta, que puede estar separada de la práctica. Si el estado-sistema es el instituidor del sistema de documentación, la búsqueda por el documento expone la forma en que el usuario del autobús se comprende a sí mismo y al Estado. $\mathrm{Al}$ decir que no es nadie y esperar ser transformado en alguien por la documentación, la persona sin documento verbaliza su noción de Estado-idea, el ente que, por medio de sus prácticas, pero principalmente sus representaciones - los funcionarios de la Justicia Itinerante, el registro de nacimiento-, puede convertirla en alguien. Al hablar de sí mismo como alguien que no existe, el usuario del autobús no habla solo de sí: deja clara su acepción sobre quién tiene el poder para transformar su existencia sí él, el Estado, por medio de la documentación.

\section{La vergüenza de Rita}

Rita es una mujer negra de 32 años y madre de cuatro hijos de 8, 10, 15 y 20 años. Auxiliar de cocina en una pizzería y diarista, moradora de Madureira, relató que respondía sola por el sustento de la casa, pues hacía años no vivía más con su excompañero. Dijo que recibía en la pizzería cerca de 900 reales mensuales, 
más lo que ganaba como señora de limpieza (150 reales por día de trabajo). Estos trabajos eran realizados en la informalidad, ya que Rita, sin registro, no tenía carnet de trabajo ni ningún otro documento. La renta se completaba con pequeños trabajos hechos por los dos hijos mayores. Ni Rita ni los hijos tienen documentos, y por eso ella buscó el autobús, aconsejada por el sacerdote de su comunidad. Mientras espera, Rita me cuenta que nunca tuvo documentos, no fue a la escuela ni votó. Según ella, la búsqueda de los documentos comenzó alrededor de los 14 años, cuando fue con su madre a intentar sacar el registro, sin éxito. Aquel era su segundo paso por el autobús: en la primera, dos meses antes, fue entrevistada por la asistente social y tuvo la primera audiencia. Después de búsquedas en oficinas y en el sistema de registro de la policía, el equipo del Sepec concluyó que Rita nunca fue registrada y la convocó a la audiencia final, que tuvo lugar el día en que me dio la entrevista.

Cuando le pregunto a Rita cómo es vivir sin documentos, ella responde: "La persona (sin documento) no es nada en la vida [...], un papel en blanco. Sin documento, la gente no es nada". De nuevo se observa la idea del documento como elemento fundamental para la constitución de la identidad. Le pregunto por qué quiere los documentos y ella dice: "Voy a sacar mis documentos toditos, estudiar, abrirle una cuenta a mi hija. Estudiar. Tengo derecho a ser alguien en la vida, ¿verdad?". Rita sabe leer y escribir "solo más o menos", porque una patrona le enseñó. Una palabra comienza a repetirse en su entrevista: vergüenza, el sentimiento que manifiesta por no tener documento. ¿Por qué?, le pregunto:

Es una vergüenza para mí. Tengo vergüenza. ¿No es para tener vergüenza? Me parece una vergüenza no tener documentos. Si trabajas en un lugar, piden documentos, y no tengo. Piden mucho. En el hospital. Me fui a comprar un teléfono, piden documentos, no tengo. Quiero abrir una cuenta para mis hijos, no tengo. [Quiero pedir el] Bolsa Familia, no tengo documentos. Es una vergüenza.

Rita me dice que tiene novio hace dos años y cuatro meses, pero el muchacho no sabe que ella no tiene documentos.

No hablé nada con él. ¿Voy a hablar para qué? ¿Eso no es una vergüenza? Es una vergüenza. Estoy hace dos años y cuatro meses con él, pero nunca le he dicho que no tengo documentos. Solo voy a hablar cuando tenga mis documentos en la mano. Yo siento vergüenza. Y mucha. Es hermoso [tener el documento]. Usted va al mercado, compra, muestra el documento. Va a una tienda, muestra el documento. Ahora usted llega a una tienda... "¿Tiene documento? ¿Dónde está el carnet de identidad?". No tengo. Para mí es una vergüenza.

Las ciencias sociales han examinado el tema de la documentación como elemento de la formación de los Estados nacionales (Bourdieu, 1996b), de control del ciudadano (Foucault, 2015) y como clave para el acceso a beneficios y derechos (Peirano, 2006; DaMatta, 2002; Carrara, 1984; Santos, 1979). Carrara (1984) relata cómo Brasil adoptó, a principios del siglo $\mathrm{xx}$, la identificación civil por el método de huellas digitales del 
serbio-argentino Vucetich, lo que sacó a la luz el debate sobre la posibilidad de que el Estado tuviera control de las características de todos los ciudadanos. DaMatta (2002) explicita el papel de los documentos como forma de control del Estado-nación sobre los ciudadanos, y afirma que su origen está en la "necesidad de inventariar los recursos humanos disponibles en la sociedad, por el conteo y clasificación de sus habitantes" (DaMatta, 2002, p. 51). Y si el documento identifica y controla, su ausencia es una señal que notar. En el estudio sobre individuos enterrados sin identificación en Río de Janeiro, la ausencia de documentación es la marca del "elemento desconocido" y, por consiguiente, inmediatamente sospechoso desde el punto de vista de perspectivas médicolegales.

En la investigación de campo, observo que la ausencia de documentación es reiteradamente asociada, por los usuarios que buscan atención en el autobús, a dos dimensiones relacionadas, la vergüenza y el ser algo sospechoso, que a su vez apuntan a una característica: ser algo escondido. La entrevista de Rita es explícita: no tener documentos es ser inmediatamente asociado a algo que, supuestamente, el individuo hizo mal. Otros usuarios explicitaron ese mismo sentimiento. Destaco algunos relatos:

- "Es muy malo no tener nada, ya he ido a la comisaría porque no tengo documentos. Da vergüenza." (David, 22 años)

- "Cuando la gente llega al puesto de salud, tiene que mentir y decir que ha olvidado los documentos. Creen que la gente hizo algo malo. Es una vergüenza, ¿verdad?". (Marta, 17 años)

- "Usted quiere ir a un lugar, no puede. Es una vergüenza. Me preguntan por qué no hice el servicio militar, como si fuera culpa mía." (Daniel, 25 años)

- "No podemos trabajar. Creen que la gente hizo algo mal. Tengo vergüenza de no tener documentos." (Raiane, 20 años)

Las percepciones de estos usuarios conducen al análisis de De Mesquita Ferreira, es decir, a la asociación de la ausencia del documento a un elemento sospechoso, asociación que se transforma en castigo en el relato de David: ir a la comisaría por no tener documentos, práctica común todavía hoy. De la misma manera, así como no tener registro imposibilita que el individuo ejerza otras actividades - como votar o, para los hombres, prestar servicio militar-, algunos usuarios del autobús relataron que terceros pagaron por ellos, en instancias variadas, por no tener documentos. No tener documentos aparece como un "problema personal", un asunto que el individuo no resolvió porque no quiso, y este se siente culpable por ello.

\section{Síntesis de los resultados}

En busca de una síntesis de los resultados, es posible destacar algunas ideas clave que atraviesan los relatos obtenidos en la investigación etnográfica. 
Tabla 1. Ideas clave de los relatos

\begin{tabular}{ll}
\hline \multicolumn{1}{c}{ Ideas clave } & \multicolumn{1}{c}{ Relatos } \\
\hline Persona sin documento = persona sin valor. & $\begin{array}{l}\text { "Un nadie", "La persona no existe", "Una nada", } \\
\text { "Una persona que no existe", "Un cero a la } \\
\text { izquierda", "Un perro". }\end{array}$ \\
$\begin{array}{l}\text { No tener documento = vergüenza, algo para } \\
\text { mantenerse en secreto. }\end{array}$ & $\begin{array}{l}\text { "Me parece una vergüenza no tener } \\
\text { documento", "No hablé nada con él [el novio]". }\end{array}$ \\
$\begin{array}{l}\text { La persona sin documento se siente culpable por } \\
\text { no tener los papeles en orden. }\end{array}$ & $\begin{array}{l}\text { "Ya he ido a la comisaría porque no tengo } \\
\text { documentos", "Creen que la gente hizo algo } \\
\text { malo", "Como si fuera culpa mía". }\end{array}$ \\
$\begin{array}{l}\text { A pesar de la concepción de sí como una persona } \\
\text { sin valor, culpable de no tener papeles, quien } \\
\text { no tiene documento también cree que merece } \\
\text { derechos, aunque estos le hayan sido negados } \\
\text { hasta ahora. }\end{array}$ & $\begin{array}{l}\text { "Yo también tengo derechos", "Tengo derecho } \\
\text { de ser alguien en la vida, iverdad?". }\end{array}$ \\
\hline
\end{tabular}

Elaboración propia

Es posible percibir, en los relatos de las usuarias y los usuarios, una dimensión moral en la que se revela un doble juicio: lo que hacen de sí por no tener documentos y lo que, a lo largo de la vida, recibieron de los demás. Las personas sin documentos hablan de sí como desprovistas de derechos, como alguien que no tiene importancia. Expresiones como "vergüenza" o "hacer algo mal" muestran esa dimensión moral curiosamente accionada en un espacio de formalidad, pues la Justicia Itinerante es una representación del Estado-sistema. En un estudio etnográfico sobre personas que buscan su registro en el programa Bolsa Familia, programa de distribución de renta del Gobierno brasileño, Marins (2014) observa que las relaciones entre beneficiarios y no beneficiarios del programa se fundan en un entramado moral, con chismes, juicios y juicios controles sociales específicos en torno a quien quiere ser incluido en el Bolsa Familia. Los beneficiarios relatan experiencias de constreñimiento, humillación y preconceptos errados por buscar acceso al programa.

Claramente, en los relatos de los usuarios del autobús, hay una matriz moral en los cobros que sufren por no tener documentos; estos responden a situaciones que van desde constreñimientos concretos (ser llevado a la comisaría) hasta soluciones momentáneas para resolver un problema -por ejemplo, el hecho de que Marta mienta en el puesto de salud al afirmar que olvidó 
los documentos, porque si decía que no los tenía, la atención era más difícil o tal vez no sucedía-. Son situaciones que no necesariamente implican una punición determinada, pero de igual forma está el sentimiento difuso de vergüenza, de haber hecho algo mal. Como indica Marta: "Hallan pronto que la gente hizo algo malo". La dimensión moral se traduce en la vergüenza que inhibe y trastorna la vida y los sentimientos.

\section{Discusión: ¿el documento como redención?}

La oficina del Juzgado de la Infancia y de la Juventud abre a las 11 de la mañana y el pasillo del edificio va siendo ocupado poco a poco por personas salidas de las audiencias en el autobús de la Plaza Once. Ellas atraviesan el patio y esperan la llegada de la funcionaria encargada de hacer el registro de nacimiento. En septiembre del 2016, la responsable es Luisa, una estudiante de historia que demuestra interés por mi investigación. Luísa recibe la decisión judicial sobre los procesos y anota en los libros notariales el registro del nacimiento de cada persona, para luego emitir el certificado de nacimiento. Va hasta el pasillo, llama al usuario por el nombre y lee el documento con él, verificando los datos. Si son correctos, entrega el documento y orienta sobre su uso: "Aquí está su certificado, no puede perderlo ni plastificarlo". Muchas veces es abrazada por los usuarios en señal de gratitud.

A lo largo de esta investigación, acompañé muchas entregas de certificados de nacimiento, entre ellas las de Rita, Cristiane y su hija Krícia. Mientras Luisa lee los datos, Cristiane empieza a llorar. Hay un error en el nombre del padre de Krícia, y el documento será rehecho. Minutos después, Luisa vuelve con el documento correcto y repite: "Aquí está su certificado, no puede perderlo ni plastificarlo". Ahora es Krícia quien llora. Rita es solo sonrisas al recibir el documento. Le pregunto qué hará en adelante.

Ahora me voy a sentir viva, ¿verdad? Con el registro en la mano. Mi mayor sueño era tener documentos, certificado. Ningún documento existe aún. Mientras no lo logre, no me tranquilizaré. Quiero estudiar, firmar mi carnet, abrir una cuenta, sacar el Bolsa Familia para mis hijos, que tengan derecho. Quiero la pensión de mis hijos.

La investigación de campo en el autobús de la Justicia Itinerante permite reflexionar sobre el registro de nacimiento a partir del concepto de rito de institución presente en Bourdieu (1996a). En diálogo con el estudio de Van Gennep sobre los ritos de pasaje, Bourdieu formula la idea de rito de institución como un proceso que modifica la condición de la persona pasa por él, pues adquiere un nuevo estatus. El rito es la mediación que consagra, legitima y hace natural lo que en verdad es arbitrario. Es un efecto casi mágico, dice, y lo que el rito "declara" se vuelve verdad (Bourdieu, 1996a). Este cambio de estatus hace que el autor decida designar tales ritos como "de institución", pues instituyen líneas, límites que separan a quien pasó por ellos de las 
personas que no los vivenciaron. Esta línea consagra una diferencia entre los dos grupos, y atribuyendo una nueva identidad a quien pasó por el rito.

El acto de institución es un acto de comunicación de una especie particular: notifica a alguien su identidad, tanto en el sentido de que él la expresa y la impone ante todos ("kategoresthat" significa, originariamente, acusar públicamente), o le notifica así con autoridad lo que ese alguien es y lo que debe ser. (Bourdieu, 1996, p. 101)

Bourdieu reflexiona sobre el papel de los nombres propios constitutivos de las identidades: el nombre es un dispositivo que actúa como marcador individual y único, "que integra la identidad del individuo en todos los campos posibles donde este interviene como agente, es decir, en todas sus historias de vida posibles" (Bourdieu, 1996a, p. 186). De Mesquita Ferreira (2009), en un estudio etnográfico sobre personas enterradas sin identificación, y Brasileiro (2008), en un análisis sobre el subregistro de niños, recurren al concepto de rito. Busco fundamentación teórica y metodológica en esta línea de trabajos para afirmar que el registro de nacimiento puede ser entendido como un rito de institución, pues es por el registro que el individuo es insertado en la sociedad. El registro identifica, define pertenencia, confiere derechos y atribuye, a quien lo recibe, el deber de mantenerse documentado en una sociedad que exige papeles. Y, porque es rito de institución, el documento separa, con una línea, a quien tiene documentos de quien no los tiene.
Una vez más, la idea de Estado como ente (Abrams, 2006) ayuda a problematizar el momento en que la persona recibe su certificado de nacimiento. De la misma manera que la falta de documentación es asociada por los usuarios a su desconstitución como sujeto de derechos ("nadie", "cero a la izquierda" $\mathrm{y}$ "persona que no existe"), la llegada del documento es relatada como la redención de todos los problemas, percibida en afirmaciones como la de Rita, que dice sentirse "viva", y de varios otros usuarios. Destaco la reacción de Damiana, una mujer negra, analfabeta, madre de cinco hijos, todos como ella, sin registro de nacimiento. Al recibir el certificado, Damiana se reía y decía: "Ahora yo voy a vivir la vida".

Porque es rito de institución, el registro de nacimiento confiere a Rita, Cristiane y Damiana un estatus que nunca tuvieron y las separa del conjunto de personas adultas sin documentación. Hasta entonces, habían vivido en los márgenes del Estado. ¿Sería el documento capaz de garantizar su salida de esos márgenes? A partir de él, podrían sacar otros documentos y solicitar el Bolsa Familia para sus hijos, y sus relatos explicitan la expectativa de que el registro pueda reconstruir existencias vividas a orillas del Estado, lo que en términos concretos significa empleo sin carnet y educación precaria, cuando no inexistente. El documento, como han explicitado varios autores, es percibido por ella como clave para acceder a derechos, beneficios y servicios, a la ciudadanía ya la afirmación. 
Un año después, no pude encontrar a Rita para saber lo que le pasó a ella. Tuve más suerte con Cristiane, y volví a entrevistarla el 18 de agosto del 2017. Trabaja en la misma casa, como cuidadora de ancianos, y cuenta que, con el certificado de nacimiento, sacó todos los documentos: CPF, carnet de identidad, título de elector y carnet de trabajo. Se inscribió en el Bolsa Familia, su objetivo inmediato cuando buscó el autobús, y ahora recibe el beneficio para la nieta. Su hija, Krícia, volvería a estudiar. "Es impresionante cómo un papel cambia la vida de la gente. Yo hasta me siento una persona mejor", me dijo Cristiane.

Volví a entrevistar a otros usuarios del autobús después de obtener el registro de nacimiento para saber cuál es el impacto del documento en sus vidas. Conseguí informaciones sobre algunos de ellos por intermedio de asistentes sociales o funcionarios públicos que los habían acompañado hasta el servicio del autobús. Aquellos con quienes conseguí hablar me trajeron detalles de cómo habían obtenido, gracias al registro, derechos y beneficios a los que nunca habían logrado acceso. Cristiane sacó el carnet de trabajo, María llevó tratamiento contra el cáncer y se jubiló, Pablo consiguió un trabajo... Ante la pregunta sobre para qué sirve un documento, mi hipótesis poco a poco se confirma: el registro de nacimiento, luego de su finalidad inmediata - conseguir otro documento, una vacante en escuela, el Bolsa Familia-, es también un documento que se transforma en clave para la ciudadanía.
Esta es una investigación todavía en curso. Pero en este artículo es posible indicar algunas conclusiones:

- Para brasileñas y brasileños sin documentos, la ausencia del registro de nacimiento está asociada a una larga trayectoria de negación de derechos, ligada a su vez a la pobreza y a la dificultad del acceso al sistema de registro. No es, como en muchos países, un problema asociado a la inmigración, sino a la desigualdad de la sociedad brasileña, donde una parte de la población tiene acceso diferenciado a derechos y ciudadanía.

- El registro de nacimiento, en su ausencia y existencia, es percibido por las usuarias y los usuarios del autobús de Justicia Itinerante como un marcador de identidad y de acceso a derechos; sin el registro, las personas hablan de sí mismas como "no personas", personas sin importancia.

- Las personas que no tienen documentos asocian esta ausencia a un sentimiento de vergüenza, con el accionamiento de matrices morales, impregnadas por la culpa.

- El estudio permite reflexionar $-y$ esta es una de las indicaciones de estudio de la tesis- sobre el papel de la Justicia Itinerante como productora de política pública; este es uno de los puntos en los que la investigación se pretende original y relevante, en tanto colabora con 
la elaboración de políticas públicas para el sector de documentación.

La conclusión final para este artículo es que es posible afirmar que el registro de nacimiento se constituye como rito de institución, capaz de modificar la condición de la persona que lo recibe. Tal modificación es percibida por las personas y por ellas expresada en sus discursos. De la entrevista de Cristiane, por ejemplo, llamo la atención sobre el hecho de que ella dice sentirse "una persona mejor". Después de todo, ¿no es ella la misma persona? De nuevo, Bourdieu ayuda en la reflexión cuando dice que el rito de institución contiene una magia performativa: "hágase lo que eres", he aquí la fórmula que subyace la magia performativa de todos los actos de institución. Para Cristiane, María y Pablo, el registro de nacimiento representó un paso en el camino para que obtengan un reconocimiento que hasta entonces les había sido negado: el de ciudadanos.

\section{Referencias}

Abrams, P. (2006). Notes on the difficult of studying the state. En A. Sharma y A. Gupta (Eds.), The anthropology of the state: a reader (pp. 112-130). Oxford: Blackwell Publishing.

Brasileiro, T. (2008). "Filho de": um estudo sobre o subregistro de nascimento na cidade do Rio de Janeiro (tesis para optar el grado de doctor en Educación). Pontifícia Universidade Catolica do Rio de Janeiro. Recuperado de http://livros01. livrosgratis.com.br/cp076371.pdf

Becker, H. (1997). Métodos de pesquisa em ciências sociais. São Paulo: Hucitec.

Boas, F. (1943). Recent anthropology. Science, (98), 334-337.

Bourdieu, P. (1996a). A economia das trocas linguísticas. São Paulo: Edusp.

Bourdieu, P. (1996b). Razões práticas: sobre a teoria da ação. Campinas: Papirus.

Brulon, V., y Peci, A. (2013). Organizações públicas e espaços às margens do Estado: contribuições para investigações sobre poder e território em favelas. Revista de Administração Pública, 47(6), 1497-1517. doi:10.1590/\$0034-76122013000600008

Carrara, S. (1984). A "sciência" e doutrina da identificação no brasil ou do controle do eu no templo da técnica. Boletim del Museu Nacional, (50), 1-27.

Caplan, J., y Torpey, J. (orgs.). (2001). Documenting individual identity. Nueva Jersey: Princeton University Press.

DaMatta, R. (2002). A mão visível do Estado: notas sobre o significado cultural dos documentos. Anuário Antropológico, (99), 37-64.

Das, V., y Poole, D. (2004). Anthropology in the margins of the state. Nuevo Mexico: School of American Research. 
Da Escóssia, F. (8 de diciembre del 2018). A fila dos invisíveis. O Globo, p. 8.

Foucault, M. (2015). Microfísica do poder. Río de Janeiro: Paz e Terra.

Garrido, B., Leonardos, L. (2017). O movimento que está sacudindo o Brasil para promover o registro civil de nascimento. En Ministério Público do Estado do Rio de Janeiro (Ed.), Acesso à documentação (pp. 23-28). Río de Janeiro: Ministério Público do Estado do Rio de Janeiro.

Hernández, A. (2010). “No dejes que la realidad...”. Apuntes sobre la representación discursiva de las personas inmigradas en los incidentes de Roquetas 2008. En M. Martínez Lirola (Ed.), Migraciones, discursos e ideologías en una sociedad globalizada. Claves para su mejor comprensión (pp. 43-56). Alicante: Instituto Alicantino de Cultura Juan Gil-Albert.

Hunter, W., y Sugiyama, N. (2017). Making the newest citizens: achieving universal birth registration in contemporary Brazil. The Journal of Development Studies, 54(3), 397-412. doi:10.1080/00220388.2017.1316378

Instituto Brasileiro de Geografia e Estatística. (2015). Estatísticas do Registro Civil. 2015. Recuperado de www.ibge.gov.br

Langness, L. (1973). A história de vida na ciência antropológica. São Paulo: EPU.

Makrakis, S. (2000). O Registro Civil no Brasil (tesis para optar el grado de magíster en Administración Pública). Fundación Getulio Vargas. Recuperado de http:// bibliotecadigital.fgv.br/dspace/bitstream/handle/10438/3405/Dissertacoes_ Solange.pdf?sequence=1\&isAllowed $=\mathrm{y}$

Marins, M. (2014). Repertórios morais e estratégias individuais de beneficiários e cadastradores do Bolsa Família. Sociologia \& Antropologia, 4(2), 543-562.

Martínez Lirola, M. (2008). Inmigración, discurso y medios de comunicación. Alicante: Instituto Alicantino de Cultura Juan Gil-Albert.

Martínez Lirola, M. (2014). Legitimating the return of immigrants in Spanish media discourse. Brno Studies in English, 40(1), 129-147.

Martínez Lirola, M. (2017). Linguistic and visual strategies for portraying immigrants as people deprived of human rights. Social Semiotics, 27(1), 21-38.

Mesquita Ferreira de, L.-d.-C. (2009). Dos autos da cova rasa. A identificação de corpos nãoidentificados no Instituto Médico-Legal do Rio de Janeiro, 1942-1960. Río de Janeiro: FINEP/E-papers.

Mesquita Ferreira de, L.-d.-C. (2013). Apenas preencher papel: reflexões sobre registros policiais de desaparecimento de pessoa e outros documentos. Revista Mana, (9), 39-68.

Mesquita Ferreira de, L.-d.-C. (2015). Pessoas desaparecidas: uma etnografia para muitas ausências. Río de Janeiro: Universidad Federal de Río de Janeiro. 
Mintz, S. (1984). Encontrando Taso, me descobrindo. Dados. Revista de Ciências Sociais, 27(1), 45-58.

Peirano, M. (2006). ¿De que serve um documento? En M. Palmeira y C. Barreira (Eds.), Política no Brasil: visões de antropólogos (pp. 25-50). Río de Janeiro: Relume Dumará / Núcleo de Antropologia da Política / Universidade Federal do Rio de Janeiro.

Pinto, D. (2014). Um antropólogo no cartório: o circuito dos documentos. Campos, 15(1), 37-56.

Pinto, D. (2016). De papel a documento: uma reflexão antropológica sobre os procedimentos notariais. Revista Antropolítica, (41), 328-356.

Santos, W. (1979). Cidadania e justiça: a política social na ordem brasileira. Río de Janeiro: Campus.

Sharma, A., y Gupta, A. (2006) Introduction: rethinking theories of the state in an age of globalization. En The anthropology of the state: a reader (pp. 1-42). Oxford: Blackwell Publishing.

Schritzmeyer, A. L. P. (2012). Introdução do eixo direito aos direitos. En A. C. Souza Lima (coord.), Antropologia e direito: temas antropológicos para estudos jurídicos (pp. 262-270). Recuperado de https://edisciplinas.usp.br/pluginfile.php/3989768/ mod_resource/content/1/Livro\%20Antropologia\%20\%20Direito\%2C\%20 $2012 \% 20 \% 28$ miolo\%29.pdf

Schritzmeyer, A. L. P. (2015). Fios da vida: crianças abrigadas, hoje adultas, diante de seus prontuários. Vivência: Revista de Antropologia, 1(46), 93-112. Recuperado de https://periodicos.ufrn.br/vivencia/article/view/8775/6260

Souza Lima, A. C. (2013). Apresentação. En A. Vianna, O fazer e o desfazer dos direitos. Río de Janeiro: E-papers.

Szreter, S. (2007). The right of registration: development, identity registration, and social security. A historical perspective. World Development, 35(1), 67-86.

Telles, V. (2010). A cidade nas fronteiras do legal e ilegal. Belo Horizonte: FinoTraço.

Van Dijk, T. (2006). Discurso de las elites y racismo institucional. Medios de Comunicación e inmigración (pp. 11-34). Murcia: Caja de Ahorros del Mediterráneo.

Vanegas, J. (2016). La antropologia del estado desde Akhil Gupta. Universitas Humanística, (82), 463-473.

Vianna, A. (2013). Introdução: fazendo e desfazendo inquietudes no mundo dos direitos. En A. Vianna (Ed.), O fazer e o desfazer dos direitos: experiências etnográficas sobre política, administração e moralidades (pp. 15-35). Río de Janeiro: E-papers. 
